\title{
DÜBLIN
}

Technological University Dublin

ARROW@TU Dublin

\section{Switchable Windows - Spectral Transmission and Switching Times}

\author{
Philippe Lemarchand \\ Technological University Dublin, philippe.lemarchand@tudublin.ie \\ Eoin D. McLean \\ Technological University Dublin, eoin.mclean@tudublin.ie \\ Brian Norton \\ Technological University Dublin, brian.norton@tudublin.ie
}

Follow this and additional works at: https://arrow.tudublin.ie/engscheleart

Part of the Electrical and Computer Engineering Commons

\section{Recommended Citation \\ Lemarchand, P., McLean, E. \& Norton, B. (2017). Switchable Windows - Spectral Transmission and Switching Times. ISES Solar World Congress 2017 IEA SHC International Conference on Solar Heating and Cooling for Buildings and Industry, 29th Oct. - 2nd Nov. 2017, Abu Dhabi, UAE. doi:10.18086/ swc.2017.12.08}

This Conference Paper is brought to you for free and open access by the School of Electrical and Electronic Engineering at ARROW@TU Dublin. It has been accepted for inclusion in Conference papers by an authorized administrator of ARROW@TU Dublin. For more information, please contact arrow.admin@tudublin.ie, aisling.coyne@tudublin.ie,gerard.connolly@tudublin.ie.

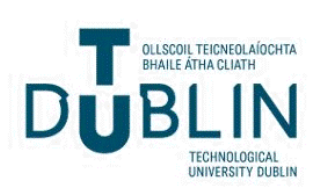




\title{
Switchable Windows - Spectral Transmission and Switching Times Philippe Lemarchand ${ }^{1}$, Eoin McLean ${ }^{1}$ and Brian Norton ${ }^{1}$ \\ 1 Dublin Institute of Technology, Dublin (Ireland)
}

\begin{abstract}
The switching time and spectral transmission in fully clear and fully opaque states of polymer dispersed liquid crystals (PDLC), suspended particle device (SPD) and electrochromic (EC) switchable windows were evaluated to assess their suitability to control solar heat transmission though glazed façade and comfort to building occupants. The transmission measurement on a SPD film showed a very effective absorption modulation in the visible range but fast decrease in the near infrared region. The switching speed was demonstrated to be related strongly to wavelengths. A SPD film with a larger absorption and faster switching speed in the infrared region was shown to be preferable to control solar heat transfer through glazings and thermal comfort. The PDLC window did not modulate transmission but rather modulated scattering. In the opaque state, the PDLC window was highly scattering resulting in spectral transmission varying at short distances from the window while being constant in the far field. The switching speed measurement demonstrated that liquid-based switchable windows can respond as fast as the eye to have the potential to control spontaneous glare. The EC window demonstrated a lower absorbance efficiency than the SPD in the visible range but appeared to have a much higher efficiency in the infrared region measured. EC windows are characterized by their slow switching speed when compared to SPD and PDLC. This makes them unsuitable for spontaneous glare control and are more suited to predictive solar heat gain control over a defined time horizon. With the increasing integration of adaptive technologies into building facades and to predict the energetical and ambient performance of such buildings and the impact on the occupants' comfort, this paper emphasises on the importance to provide spectral performance information of switchable windows.
\end{abstract}

Keywords: switchable window, spectral transmission, switching speed, adaptive façade, solar control.

\section{Introduction}

Switchable windows switch between a clear and an opaque transparent state by controlling light absorption, reflection and/or scattering. EC, liquid crystal (LC) based and electrophoretic or SPD technologies are the most promising for manufacturing large area electrically activated switchable windows for control of solar energy through building and vehicle glazings.

The coefficient of solar energy transmittance ( $g$-value) or solar heat gain coefficient (SHGC) and shading coefficient are determined knowing the incident solar insolation and the spectral transmittance of glazing over the entire solar spectrum (Geymard, 2007; Gueymard and duPont, 2009; Waide and Norton, 2003). These coefficients vary significantly with the incident solar insolation. With adaptive windows dynamically adapting their optical transmission these coefficients will vary furthermore. As visible light transmission and scattering is modulated, indoor lighting conditions vary with illuminance, colour rendering index and daylight glare probability (Wienold, 2010) the affected quantities. It is therefore important to disaggregate the impact of the visible component of the spectrum affecting indoor lighting and glare, and the infrared component of the spectrum contributing to the indoor thermal performance via solar gain and the greenhouse effect further affecting the cooling and heating loads of the building and occupants' comfort.

Switchable windows do not prescribe requirements on switching times. For building application, the short time change of glare (visible wavelength) and solar heat (infrared wavelengths) incident on a surface typically vary with the passage of watery clouds. Considering an instant change of the solar irradiance by the passing of a cloud, the required switching time for switchable windows would correspond to the human response to light. Presumably, switchable windows would need to switch as fast as the eye response, i.e. $13 \mathrm{~ms}$ to $80 \mathrm{~ms}$ (Lewis, 2014), to have a spontaneous control of the glare.

The human sensation of heat given by the heat index varies by $8^{\circ} \mathrm{C}$ when moving from shade to sunlight (NWSWFO, 
2004). Moreover, the human skin response time to cold is $0.1^{\circ} \mathrm{C} / \mathrm{s}$ (Jones, 2009) and discomfort is perceived with only $1{ }^{\circ} \mathrm{C}$ difference to body temperature which is felt in the order of 10 seconds (Kenny, 1998). Consequently, the $8^{\circ} \mathrm{C}$ difference felt from shadow to sunlight brings a sensation of discomfort in the order of 1.25 seconds which would then be the desired response time to solar heat transfer (infrared wavelength) through the switchable glazing.

Knowing the spectral transmission and switching time performances of switchable windows as further studied below can potentially enable to appropriately and more accurately control such adaptive technologies with the desired building energy performance and occupant comfort.

\section{Methodology: sample characteristics and optical setups}

\subsection{Sample characteristics}

The EC EControl ${ }^{\mathrm{TM}}$, SPD SmartGlass ${ }^{\mathrm{TM}}$, PDLC SmartGlass ${ }^{\mathrm{TM}}$ glazings as well as SPD and PDLC films (without glass panes) presented in Tab. 1 and Fig. 1 were characterized. The EC glazing was electrically switched by its controller applying between $-1 \mathrm{Vdc}$ and $+2 \mathrm{Vdc}$ measured at the window's electrodes. The window transmissions provided by the manufacturer are measured according to DIN EN 410 within the 380-780nm region. The EC window were specified to be $50 \%$ and $10 \%$ in the transparent and opaque state respectively with a tolerance of $+/-2 \%$. Both the SPD and PDLC films and glazing were electrically switched between OVac and 110Vac. Consequently, the EC and SPD films worked either in a transparent mode when turned ON or into an opaque mode when turned OFF, the window appearing tinted with a dark blue colour. The LC films were in their transparent state when turned ON, and exhibited a milky-white tint when turned $\mathrm{ON}$ and were consequently in their opaque mode. A Lambda900 spectrophotometer was used to measure the full and scattered transmission through the SPD and PDLC films, the glazings and each individual component forming the SPD and PDLC glazings. Due to the size of the EC glazing that could not fit in the Lambda900, a second dedicated optical setup was used. This setup was used to measure the optical transmission of the EC glazings and the switching time of the SPD, PDLC and EC glazings.

Tab. 1: SPD, PDLC and EC tested samples.

\begin{tabular}{|lllr|}
\hline Sample name & Shape & \multicolumn{1}{c|}{ Size } & Thickness Configuration \\
\hline SPD glazing & Flat & $\mathrm{L}=28 \mathrm{~cm}, \mathrm{H}=18 \mathrm{~cm}$ & $4 \mathrm{~mm}$ glass $/ \sim 1 \mathrm{~mm}$ space $/ 4 \mathrm{~mm}$ glass \\
PDLC glazing & Flat & $\mathrm{L}=28 \mathrm{~cm}, \mathrm{H}=18 \mathrm{~cm}$ & $4 \mathrm{~mm}$ glass $/ 2 \mathrm{~mm}$ space $/ 4 \mathrm{~mm}$ glass + silver coating \\
EC glazing & Flat & $\mathrm{L}=32 \mathrm{~cm}, \mathrm{H}=32 \mathrm{~cm}$ & $4 \mathrm{~mm}$ glass $/ \sim 1 \mathrm{~mm}$ space $/ 4 \mathrm{~mm}$ glass $/$ cavity $/ 4 \mathrm{~mm}$ low-E coated glass \\
SPD film & Flat & $\mathrm{L}=5 \mathrm{~cm}, \mathrm{H}=5 \mathrm{~cm}$ & ITO/SPD film/ITO \\
PDLC film & Flat & $\mathrm{L}=5 \mathrm{~cm}, \mathrm{H}=5 \mathrm{~cm}$ & ITO/PDLC film/TTO \\
\hline
\end{tabular}

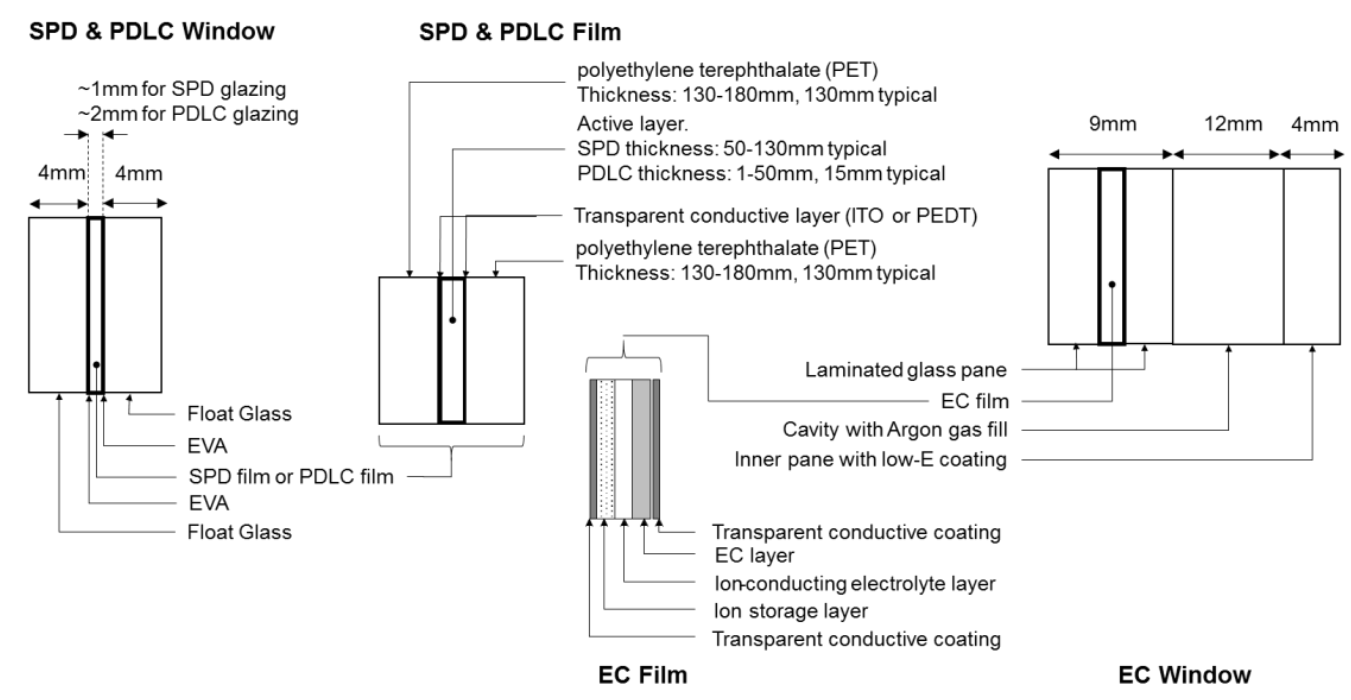

Fig. 1: Schematic of the tested SPD and PDLC films and SPD, PDLC and EC windows

\subsection{Lambda900 spectrophotometer for film transmission analysis}

Fig. 2 and Fig. 3 represent the reference beam (1) and the sample beam (2) within the Lambda900, with same optical path length $(\mathrm{D} \approx 36 \mathrm{~cm})$ from their respective cuvette holder to the integrating sphere (IS) aperture of diameter $\Phi=5 \mathrm{~mm}$. Wavelengths from $250 \mathrm{~nm}$ to $2500 \mathrm{~nm}$ were scanned in $1 \mathrm{~nm}$ steps to look at the film ultraviolet (UV), visible (VIS), near-infrared (NIR) and short-wave infrared (SW-IR) transmission and compare them with the optical bench 
setup results. Absorbance calibration was done in air. For measurement of all transmitted light, a maximum of scattered light was collected for the switchable film (SPD or LC film with ITO layers on both sides and no glass) located flat at the integrating sphere aperture entrance. All forward scattered light sketched in Fig. 4 was collected by the integrating sphere. Transmission in the far-field $(\sim 36 \mathrm{~cm}$ from the IS) was achieved by setting the film flat at the cuvette or further named "sample port" (SP). The cone angle $\theta \mathrm{t}$ of collected transmission is reduced to $\theta \mathrm{t} \approx 0.15^{\circ}$ as illustrated in Fig. 5. The cone angle in that configuration being close to $0^{\circ}$, transmission measurements are considered to correspond to the specular and direct $\left(0^{\circ}\right)$ transmissions.

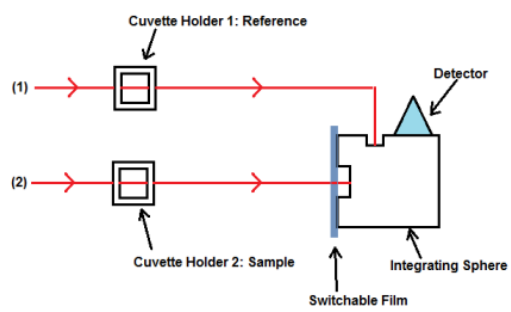

Fig. 2: Film scattered transmission measurement at the Lambda900 integrating sphere.

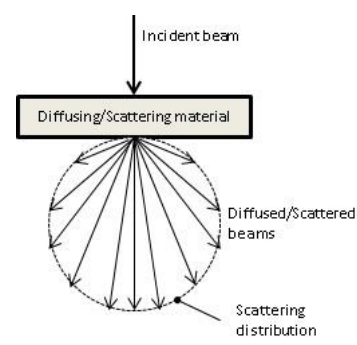

Fig. 4: Scattered transmission sketch. The doted circle represents the irradiance distribution of the scattered light.

$$
\begin{aligned}
& \eta_{\text {abs }}=\frac{T_{\text {clear }}-T_{\text {opaque }}}{T_{\text {clear }}}=1-\frac{T_{\text {opaque }}}{T_{\text {clear }}} \\
& T_{\text {scatt }}(\%)=T_{I S}(\%)-T_{S P}(\%) \\
& \alpha=\frac{\pi * D_{p}}{\lambda}
\end{aligned}
$$

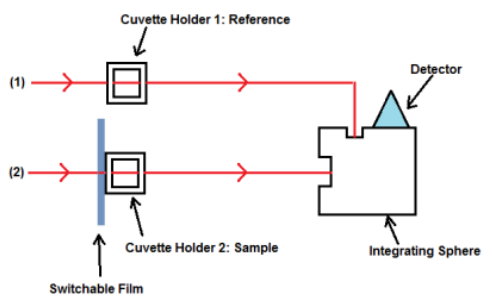

Fig. 3: Far field film transmission at the cuvette in Lambda900.

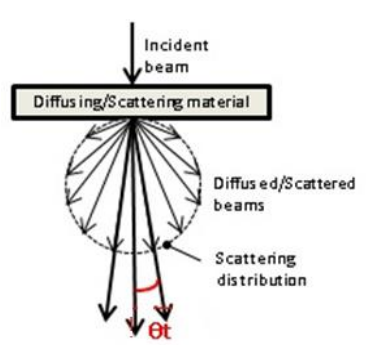

Fig. 5: Far field transmission sketch.

The dynamic light attenuation when switching ON and OFF the samples could be obtained from the transmission curves. The relative attenuation of the signal can be seen as the relative absorption efficiency $\eta_{\mathrm{abs}}$ of the film expressed by (eq. 1 . As calculated by (eq. 2, the difference between transmission measurements at the IS port ( $\mathrm{T}_{\mathrm{IS}}$ ) and sample port $\left(\mathrm{T}_{\mathrm{SP}}\right)$ provided information on the percentage transmission affected by scattering. (eq. 3 describes the scattering dimension less parameter $\alpha$ with $\mathrm{D}_{\mathrm{p}}$ the diameter of a spherical particle and $\lambda$ the wavelength. The parameter $\alpha$ enables to distinguish three types of scattering: 1) Rayleigh scattering for $\alpha<<1$; 2) Mie scattering for $\alpha \approx 1$ and 3) geometric scattering for $\alpha \gg>1$. With the incident light wavelength of the same order as the particles' sizes. The condition where $\alpha \approx$ Mie scattering $(\alpha \approx 1)$ enables to have a view of the particles size distribution across the studied film. Once the switchable film is in position, transmission measurements in clear and opaque states are separated by several minutes ( $\sim 5$ minutes) to ensure the films in their opaque state reach their thermodynamic equilibrium and are optically stable.

\subsection{Optical bench setup and its validation with float glass and EVA transmission}

A solar simulator and integrating sphere fibre connected to a handheld spectrometer was used to i) determine the absorption contribution of glass and EVA layers in the final glazing, ii) to ensure the optical bench setup results coincide with the Lambda900 spectrophotometer and iii) to measure the switching time of the EC, SPD and PDLC glazing. A first sample, named 'FGEVAFG' hereafter, comprised of an ethylene-vinyl acetate (EVA) layered in between two float glass (FG) panes $6 \mathrm{~mm}$ thick was measured in transmission using both setups. The absorption measurement of a single $6 \mathrm{~mm}$ FG pane further enabled the determination of the transmission of the EVA layer only. 
Both transmission measurements at the IS and SP of the Lambda900 were perfectly matching between $250 \mathrm{~nm}$ and $2500 \mathrm{~nm}$, demonstrating no scattering occurred. Transmission measurements compared using the optical bench setup validated the setup results between $385 \mathrm{~nm}$ and $1020 \mathrm{~nm}$ (see Fig. 6).

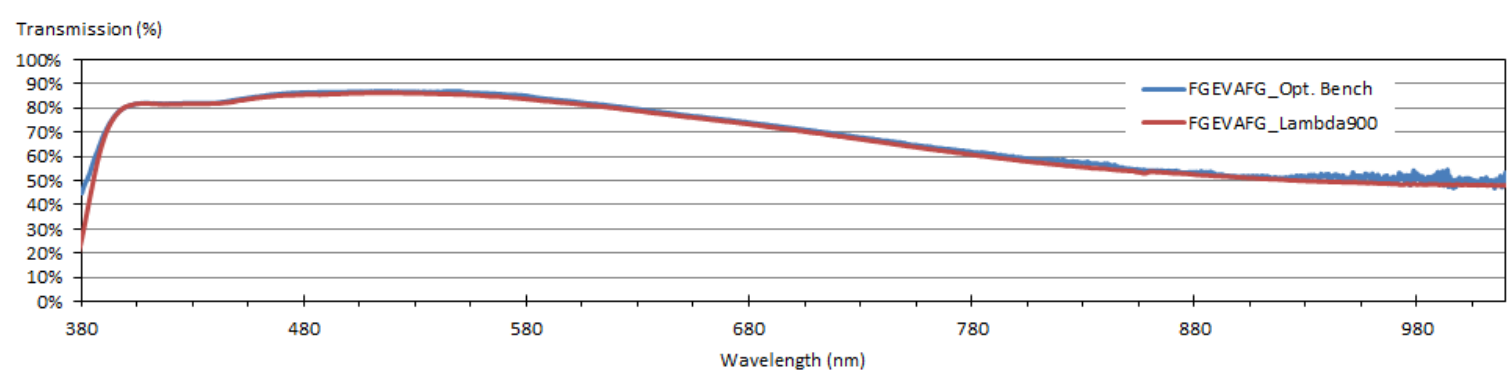

Fig. 6: FG-EVA-FG transmission and optical bench setup validation.

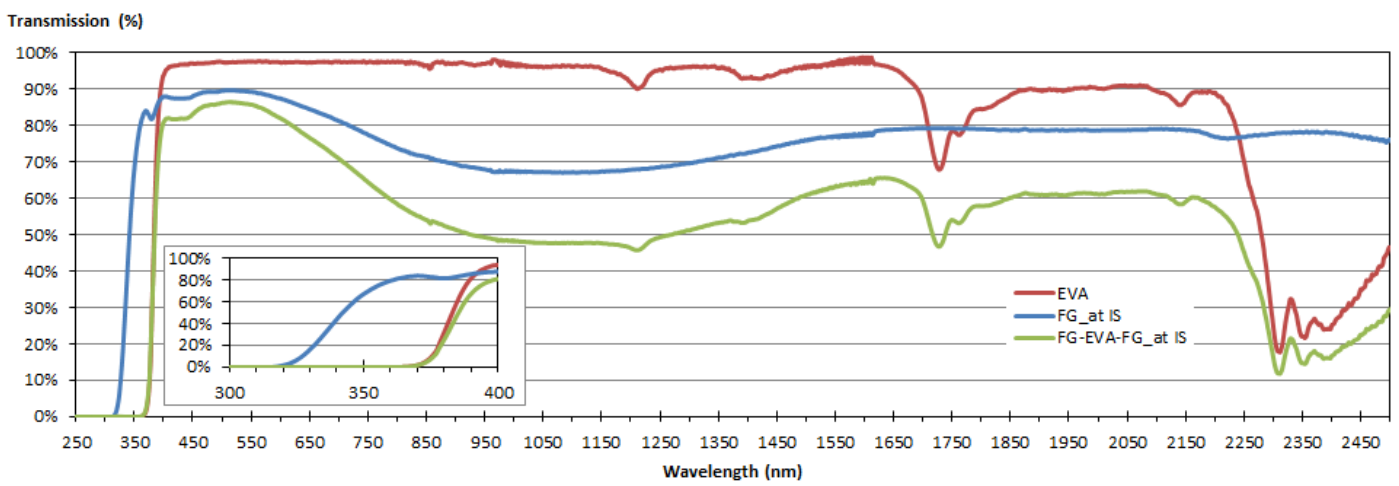

Fig. 7: Transmission of 4mm float glass (FG), FG-EVA-FG sample and EVA layer.

The absorbance $(\mathrm{Ab})$ of the float glass and the FGEVAFG sample measured, the transmission of the EVA layer was calculated using (eq. 4 .

$A b_{F G E V A F G} \approx A_{F G E V A F G}+R_{F G} \approx R_{F G}+A_{E V A}+2 * A_{F G}$ discarding multiple internal reflections $A b_{F G}=A_{F G}+$ $R_{F G}$ and $A b_{F G E V A F G}-2 * A b_{F G} \approx A_{E V A}-R_{F G}$ leading to:

$$
T_{E V A}(\%)=\frac{100}{10^{A b_{F G E V A F G}-2 * A b_{F G}}} * 10^{-R_{F G}}
$$

(eq. 4 requires the spectral reflection at the air-FG interface $R_{F G}$ approximated using the Fresnel reflection in (eq. 5 for unpolarized light (Albregtsen, 2008) and considering the known refractive index of BK7 available between 300nm and 2500nm (Polyanskiy, 2016).

$$
\begin{aligned}
R_{s}=\left|\frac{n_{1} * \cos \theta_{i}-n_{2} * \cos \theta_{t}}{n_{1} * \cos \theta_{i}+n_{2} * \cos \theta_{t}}\right|^{2}=\left|\frac{n_{1} * \cos \theta_{i}-n_{2} * \sqrt{1-\left(\frac{n_{1}}{n_{2}} * \sin \theta_{i}\right)^{2}}}{n_{1} * \cos \theta_{i}+n_{2} * \sqrt{1-\left(\frac{n_{1}}{n_{2}} * \sin \theta_{i}\right)^{2}}}\right|^{2} \\
R_{p}=\left|\frac{n_{1} * \cos \theta_{t}-n_{2} * \cos \theta_{i}}{n_{1} * \cos \theta_{t}+n_{2} * \cos \theta_{i}}\right|^{2}=\left|\frac{n_{1} * \sqrt{1-\left(\frac{n_{1}}{n_{2}} * \sin \theta_{i}\right)^{2}}-n_{2} * \cos \theta_{i}}{n_{1} * \sqrt{1-\left(\frac{n_{1}}{n_{2}} * \sin \theta_{i}\right)^{2}}+n_{2} * \cos \theta_{i}}\right|^{2} \\
R=\frac{R_{s}+R_{p}}{2}
\end{aligned}
$$

In the equations above, the subscripts ' $s$ ' and ' $p$ ' respectively denote the perpendicular and parallel electric field direction of the light electromagnetic wave. The subscripts ' $i$ ' and ' $t$ ' refer to the incident and transmitted angles $\theta$ at the air-FG interface of respective refractive index $n_{1}$ and $n_{2}$.

The resulting EVA transmission curve in Fig. 7 showed relatively flat transmissions of above 95\% from 430nm to $1650 \mathrm{~nm}$ and $\approx 90 \%$ from $1850-2200 \mathrm{~nm}$. Strong absorption bands occurred in the $1655-1750 \mathrm{~nm}$ and $2325-2400 \mathrm{~nm}$ ranges. The glazing thickness is mostly comprised of glass. The FGEVAFG sample transmission showed that the 
choice of glass highly affects the overall glazing performance- most importantly the NIR, SW-IR ranges and the consequent solar transmission. The UV cut-off wavelengths of float glass and EVA were respectively noted around $320 \mathrm{~nm}$ and $370 \mathrm{~nm}$. The EVA, absorbing UV, acts as a protective filter for particles embedded into switchable windows but UV sensitive particles would still deteriorate under long-term UV exposure (Czanderna, 1996).

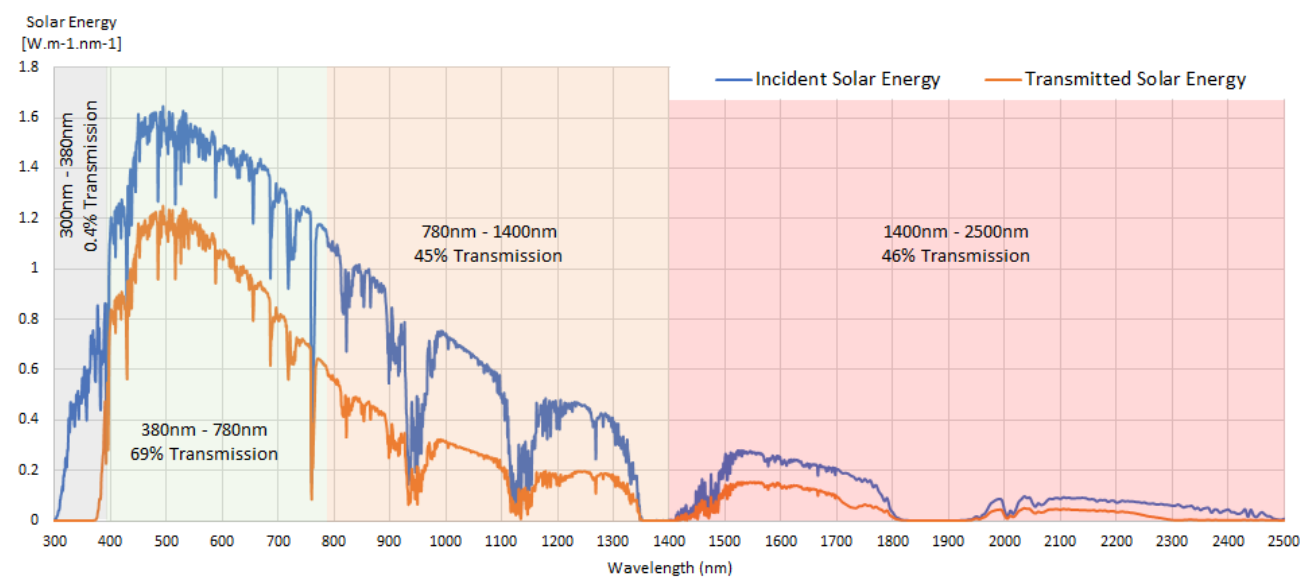

Fig. 8: Solar energy spectrum incident and transmitted through $12 \mathrm{~mm}$ of the measured clear float glass and $0.4 \mathrm{~mm}$ EVA layer.

Knowing the absorbance of the float glass and the EVA layers enabled the calculation of the Napieran attenuation coefficient of the materials and simulation of the transmission through three glass panes $4 \mathrm{~mm}$ thick and two EVA layers $0.2 \mathrm{~mm}$ thick (see Fig. 8). This configuration, similar to the EC window without the active EC film and the low-E coating, enabled an understanding of the amount of solar energy available for transmission modulation by a switchable film. The measured clear float glass showed $\sim 69 \%$ transmission in the visible and strong absorption in the NIR and SW-IR regions with transmission to $\sim 45 \%$ and $\sim 46 \%$ respectively. The transmitted solar energy was about $371 \mathrm{~W} . \mathrm{m}^{-2}, 145 \mathrm{~W} . \mathrm{m}^{-2}$ and $47 \mathrm{~W} . \mathrm{m}^{-2}$ in the respective wavelength ranges. The EVA layer absorbing UV, $0.4 \%$ transmission is calculated between $300 \mathrm{~nm}$ and $380 \mathrm{~nm}$, representing $\sim 120 \mathrm{~mW} . \mathrm{m}^{-2}$. Simulation on clearer glass such as the Planiclear ${ }^{\mathrm{TM}}$ from Saint-Gobain demonstrated transmission of about $1.2 \%, 83 \%, 63 \%$ and $63 \%$ in the UV, VIS, NIR and SW-IR respectively and therefore enabling a larger fraction of the solar energy to be modulated by an active film integrated between the two EVA layers.

\section{Spectral transmission analysis}

\subsection{SPD and PDLC films}

The SPD film tested, about $0.35 \mathrm{~mm}$ thick, is mainly made of a polymer (most likely PMMA) for which absorption bands occurred around 1657nm, 2143nm and 2328-2450nm in Fig. 9 and again for the PDLC film in Fig. 10. The transmission curves at both the IS and SP positions for the ON state were matching, meaning reflections from all internal interfaces and absorption are the only occurring phenomena. In the OFF state, particles in random orientations strongly absorb light in the visible and NIR range. The transmission curves at the IS and SP showed differences in the transmitted blue and NIR wavelength ranges. Plotting the scattered transmission (difference between the two curves), a Poisson distribution was obtained between $760 \mathrm{~nm}$ and $2250 \mathrm{~nm}$ with a maximum of the particles having a size about $1 \mu \mathrm{m}$ corresponding to the particles length (Saxe et al. 2003) and confirming this difference is attributable to a Mie scattering process ( $\alpha \approx 1$ in eq. 2 ).

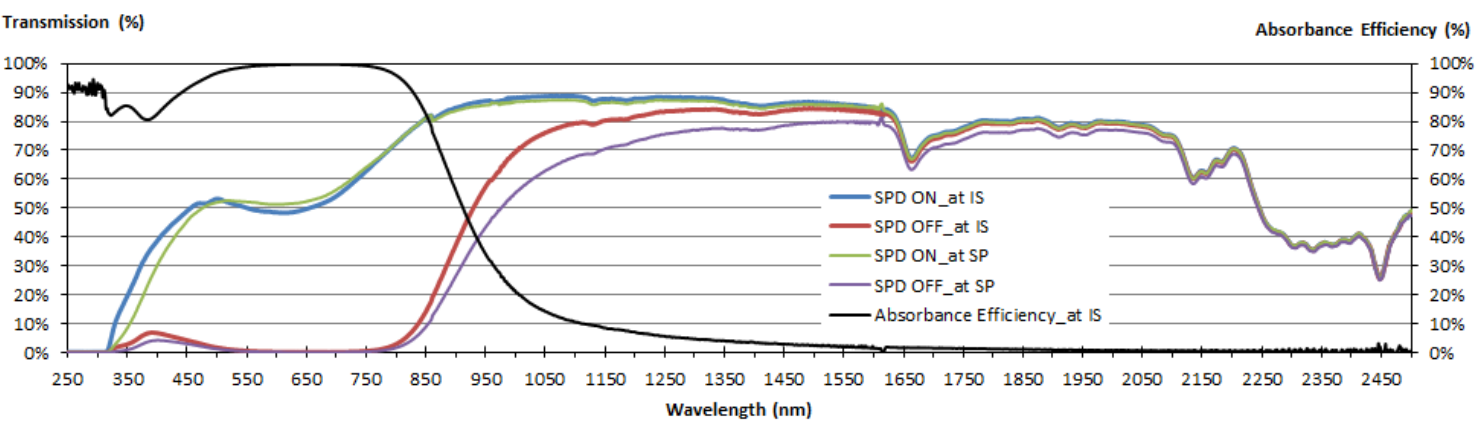

Fig. 9: SPD film transmission and absorbance efficiency 
Multiplying the transmission curve by the global solar irradiance at an air-mass of 1.5 (ASTM, 2012), the spectral solar irradiance and solar energy transmitted through the SPD film (i.e. without glazing and interfacing EVA) in the transparent and opaque states was obtained and summarised in Tab. 2.

Tab. 2: Global solar energy modulated by the SPD film.

\begin{tabular}{|c|c|c|c|c|c|}
\cline { 3 - 4 } \multicolumn{2}{c|}{} & \multicolumn{2}{c|}{ Transmitted solar energy $\left(\mathrm{W} / \mathrm{m}^{2}\right)$} & \multicolumn{1}{c|}{$\begin{array}{c}\text { Solar energy } \\
\text { modulated }\left(\mathrm{W} / \mathrm{m}^{2}\right)\end{array}$} & $\begin{array}{c}\text { Fraction of solar } \\
\text { energy modulated }(\%)\end{array}$ \\
\hline $\begin{array}{c}\text { Spectral } \\
\text { range }(\mathrm{nm})\end{array}$ & $\begin{array}{c}\text { Global solar } \\
\text { energy } \\
\left(\mathrm{W} / \mathrm{m}^{2}\right)\end{array}$ & Transparent & Opaque & 5.4 & $16.9 \%$ \\
\hline $280-379.5$ & 32.1 & 6.5 & 1.1 & 256.6 & $49.6 \%$ \\
\hline $380-780$ & 535.5 & 274.0 & 8.4 & 106.1 & $32.8 \%$ \\
\hline $781-1400$ & 323.8 & 272.8 & 166.7 & 1.2 & $1.1 \%$ \\
\hline $1401-2500$ & 101.9 & 76.0 & 74.9 & 378.3 & $38.1 \%$ \\
\hline $280-2500$ & 993.3 & 629.4 & 251.1 & & \\
\hline
\end{tabular}

Fig. 9 shows the SPD window highly modulated transmission in the wavelength range from $390 \mathrm{~nm}$ to $820 \mathrm{~nm}$ with transmission varying from $50 \%$ to $70 \%$ above $450 \mathrm{~nm}$ and averaging at $51.2 \%$ transmission over the visible range (380nm-780nm). The SPD showed a strong absorption from $1000 \mathrm{~nm}$ to $2100 \mathrm{~nm}$ with a modulation efficiency continuously decreasing below $30 \%$ from above $1000 \mathrm{~nm}$ and below $10 \%$ above $1350 \mathrm{~nm}$. In Tab. 2, the SPD film appeared to modulate $16.9 \%$ of the UV light $(280 \mathrm{~nm}-380 \mathrm{~nm})$ with $6.5 \mathrm{~W} \cdot \mathrm{m}^{-2}$ of solar UV transmitted in the transparent state. Considering the $\sim 120 \mathrm{~mW} . \mathrm{m}^{-2}$ transmitted through the simulated glazing, between $4 \mathrm{~mW} . \mathrm{m}^{-2}$ and $24 \mathrm{~mW} . \mathrm{m}^{-2}$ is transmitted in the opaque and transparent modes. To mitigate degradation of the SPD film, exposed to $\sim 850 \mathrm{~mW} . \mathrm{m}^{-2}$ after $4 \mathrm{~mm}$ glass and $0.2 \mathrm{~mm}$ EVA layer, or other materials within the occupant environment, UV protection is initially achieved with float glass and EVA layers forming the window and would benefit from the potential addition of a UV filter. In the transparent state, about half of the solar energy in the visible range is absorbed which significantly limits the fraction of natural light entering the occupant environment on cloudy days. With $49.6 \%$ of visible solar energy controllable, this is the spectral band most effectively controlled by the SPD film. In the nearinfrared (781-1400nm) the SPD still modulated $32.8 \%$ of the input solar energy but a high $166.7 \mathrm{~W} . \mathrm{m}^{-2}$ solar energy was transmitted in the opaque state and represented more than $51.4 \%$ of the solar energy in that range. This implies that there is significant infra-red solar heat transfer in the switched-mode that is ostensibly intended to reduce solar gain. SPD materials with an absorption efficiency in the NIR similar to current performances within the visible range could lead to an additional $16.8 \%$ to the current modulation capability. The latter represents an additional $51.2 \%$ of energy modulable through a glazing integrated the active film. For the simulated glazing with $12 \mathrm{~mm}$ glass thickness and $0.4 \mathrm{~mm}$ EVA layer, it represents an additional $4.3 \%$ solar (280-2500nm) energy modulation compared to $4.9 \%$ for a clearer glass such as the Planiclear ${ }^{\mathrm{TM}}$. Considering the film thickness and the low dynamic absorption of SPD material above $1400 \mathrm{~nm}$, only $1.1 \%$ of the solar energy in the short-wave infrared $(1400-2500 \mathrm{~nm})$ could be modulated with more than $73 \%$ of the solar energy transmitted through the SPD independently of the SPD optical state. The $780 \mathrm{~nm}-2500 \mathrm{~nm}$ solar range containing $42.6 \%$ of the $1 \mathrm{~kW} \cdot \mathrm{m}^{-2}$ global solar energy and $\sim 34 \%$ of the solar energy transmitted through the simulated window, innovative SPD materials with an efficient dynamic control of the infrared range would significantly contribute to the infrared solar heat transfer modulation offered by switchable windows. Overall, in the $280-2500 \mathrm{~nm}$ range, the SPD film could modulate $38.1 \%$ of the solar energy passing through a glazing

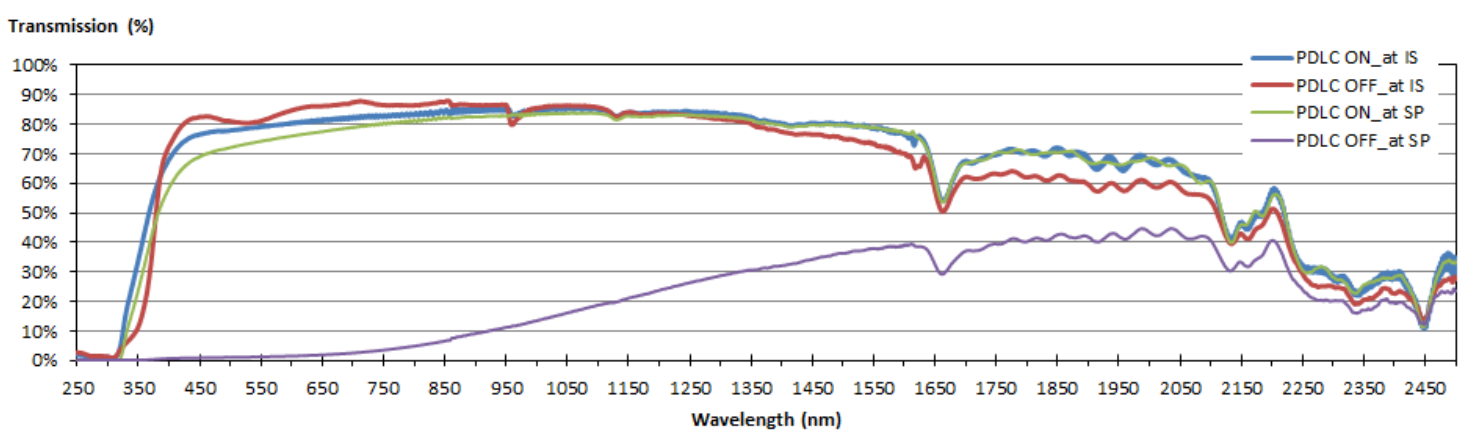

Fig. 10: PDLC film transmission.

Transmission results on the PDLC film shown in Fig. 10 demonstrated very similar transmission in the clear and opaque state over the entire scanned range when measured at the integrating sphere. This result led to conclude that light passing through the film is not further absorbed nor significantly more reflected when switching ON or OFF the film. However, when setting the film at the sample port, transmission is highly reduced in the OFF state meaning 
that 1) almost all visible light passing through the film in the OFF state is scattered (giving the milky-white appearance) and 2) that the transmission loss mechanism in the OFF state is uniquely related to the distance between the detector and the film until the cone angle becomes small enough (close to 0 radian) that the transmission will not change anymore.

\subsection{EC window transmission}

The EC window transmission curves for both the opaque and transparent states are shown in Fig. 11 together with the absorbance efficiency. In the transparent state, the EC window tested, demonstrated transmission in the visible (380-780nm), near-infrared (780-1020nm) and solar (380-1020nm) ranges of 50.1\%, 50.8\% and 29.2\% respectively. In the opaque state, the EC window transmission went down to $15.3 \%, 14.9 \%$ and $0.8 \%$ at the respective range. The transmission tolerance in those ranges were $\pm 1.0 \%, \pm 1.1 \%$ and $\pm 4.9 \%$ due to the intensity of the source, the fluctuation of the lamp, the sensitivity of the spectrometer and the signal noise in the various wavelength ranges. While the $50.1 \%$ visible transmission in the transparent state of the window was matching the manufacturer information, the $15.3 \pm 1.0 \%$ transmission in the opaque state was not matching the $10 \pm 2 \%$ transmission. A possible cause of this, suggested by the manufacturer, was the lack of sunlight exposure of the glazing and switching in the time leading up to the test. The EC glazing requires UV sunlight exposure to allow it to fully darken. This encourages further understanding of the influence of outdoor solar conditions and temperature on the glazing transmission and switching speed capabilities. In the visible range the window has an absorbance efficiency varying from about $70 \%$ at $380 \mathrm{~nm}$ down to $50 \%$ at $480 \mathrm{~nm}$ before rising to nearly $95 \%$ at $780 \mathrm{~nm}$. The signal noise in the opaque mode did not allow a clear transmission measurement above $880 \mathrm{~nm}$ but it appeared the window absorbance efficiency tends to $100 \%$ up to $1020 \mathrm{~nm}$ likely due to the low-E reflective coating in the fifth glass surface of the window. A measurement of the EC film only using the Lambda900 is desirable to have a higher accuracy of results over the entire solar range.

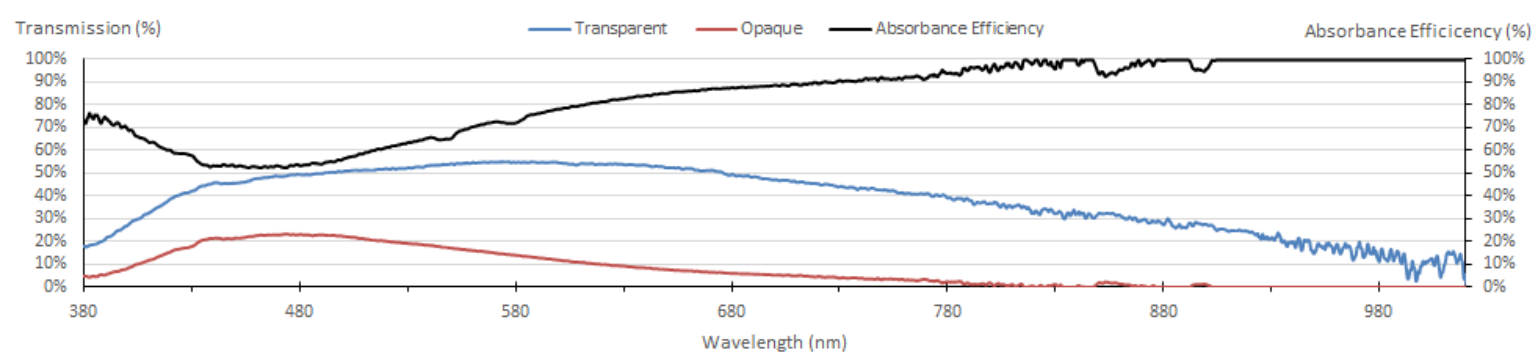

Fig. 11 EControl EC Window - Transmission in the transparent and opaque states and absorbance efficiency

Similar to the SPD film, the global solar irradiance transmitted through the EC window in the transparent and opaque states was obtained in Fig. 12 and the solar energy summarised in Tab. 3.

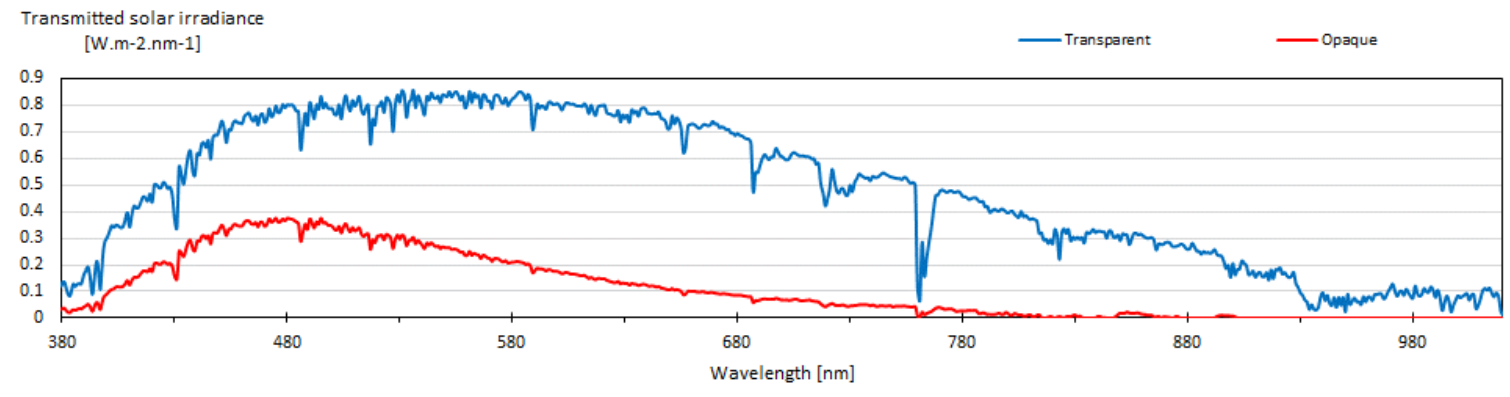

Fig. 12: Calculated solar irradiance transmitted through the EC window in the transparent and opaque states

Tab. 3: Global solar energy modulated by the EC window.

\begin{tabular}{|c|c|c|c|c|c|}
\hline \multirow[b]{2}{*}{$\begin{array}{c}\text { Spectral } \\
\text { range }(\mathrm{nm})\end{array}$} & \multirow[b]{2}{*}{$\begin{array}{c}\text { Global solar } \\
\text { energy } \\
\left(\mathrm{W} / \mathrm{m}^{2}\right)\end{array}$} & \multicolumn{2}{|c|}{ Transmitted solar energy $\left(\mathrm{W} / \mathrm{m}^{2}\right)$} & \multirow[b]{2}{*}{$\begin{array}{c}\text { Solar energy } \\
\text { modulated }\left(\mathrm{W} / \mathrm{m}^{2}\right)\end{array}$} & \multirow[b]{2}{*}{$\begin{array}{c}\text { Fraction of solar } \\
\text { energy modulated }(\%)\end{array}$} \\
\hline & & Transparent & Opaque & & \\
\hline $380-780$ & 535 & $258 \pm 6$ & $70 \pm 6$ & 188 & $35.1 \%$ \\
\hline $780-1020$ & 188 & $50 \pm 9$ & $1 \pm 9$ & 49 & $26.1 \%$ \\
\hline $380-1020$ & 723 & $308 \pm 8$ & $71 \pm 8$ & 237 & $32.8 \%$ \\
\hline
\end{tabular}

Similar to the SPD film in the transparent state, Tab. 3 shows that the EC window transmission in the visible range 
significantly limits the fraction of natural light entering the occupant environment on cloudy days. With $35.1 \%$ of visible solar energy controllable, this is the spectral band most effectively controlled. In the near-infrared (780$1020 \mathrm{~nm}$ ) the fraction of solar energy modulated goes down to $26.1 \%$ with about $50 \mathrm{~W} . \mathrm{m}^{-2}$ solar energy transmitted in the transparent state and $0-10 \mathrm{~W} . \mathrm{m}^{-2}$ in the opaque state. Such low transmission in the infrared in both states is the result of the film light absorption and the light reflection from the low-E coating on the fifth glass surface. Such configuration would allow the window to significantly reject infra-red solar heat transfer but still be able to modulate a small portion of the solar energy in that region. Thus, it is convenient for climates with a high solar irradiance. In low to moderate solar irradiance conditions, the window would benefit from modulating a larger fraction of the solar range as more visible light and infrared solar heat passing through the window might be required. Overall, the EC window could control $32.8 \%$ of the solar energy contained within the $380 \mathrm{~nm}-1020 \mathrm{~nm}$ range.

\section{Switching time of SPD, PDLC and EC windows}

In principle, the complete switching time between fully clear to fully opaque is identical at all wavelengths due to the time it takes for the SPD particles and LC molecules to rotate or the ions to be fully absorbed by the EC layer according to the voltage applied. However, the relative spectral switching time further on called "switching time" varies according to the intensity of the source and the spectral transmission in the transparent and opaque states at the wavelength considered. Using the optical bench setup, the switching time of the SPD, PDLC and EC windows could be measured. When switching the windows, the light transmission change with time, was observed rising and decreasing similar to the charging and discharging curves of an electronic Resistor-Capacitor (RC) circuit. As illustrated in Fig. 13, the time $\tau$ was defined as the time required for the window to reach $63 \%$ of its final state (transparent or opaque). The defined switching time $3 \tau$ is the time required to reach $95 \%$ of the transition and $5 \tau$ for $99 \%$ of the transition.
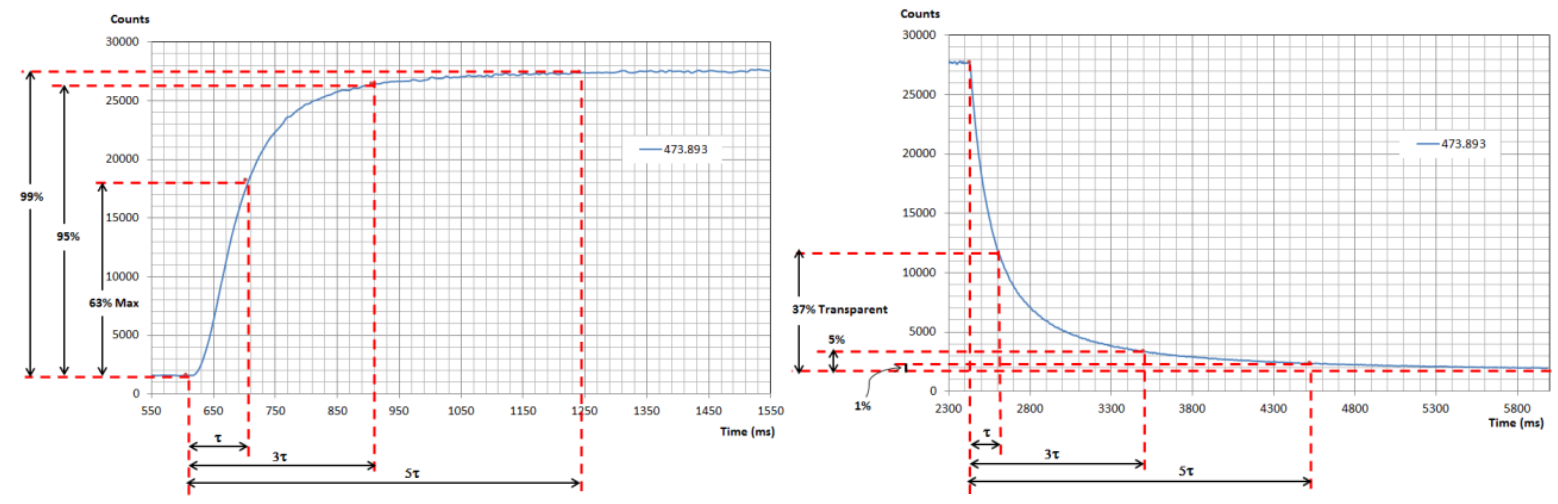

Fig. 13: (left) Switching ON time $\left(\tau_{\text {up }}\right)$ and (right) switching OFF time $\left(\tau_{\text {down }}\right)$ definition. Examples with the SPD glazing at 473nm.

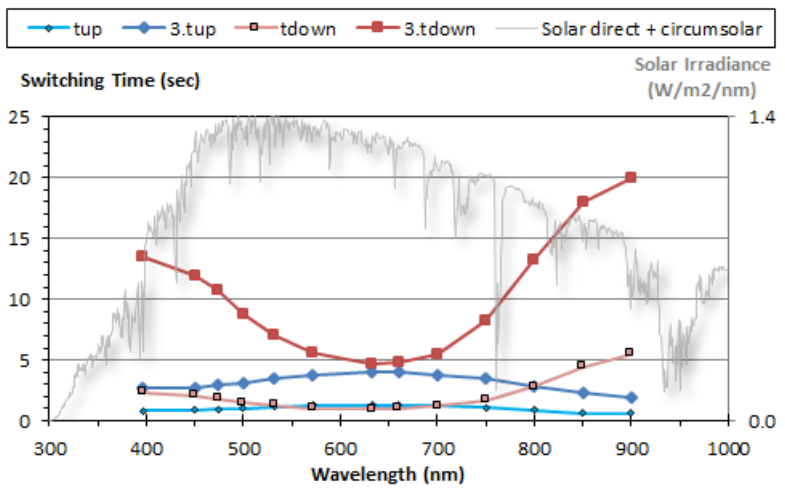

Fig. 14: SPD window switching ON and OFF times.

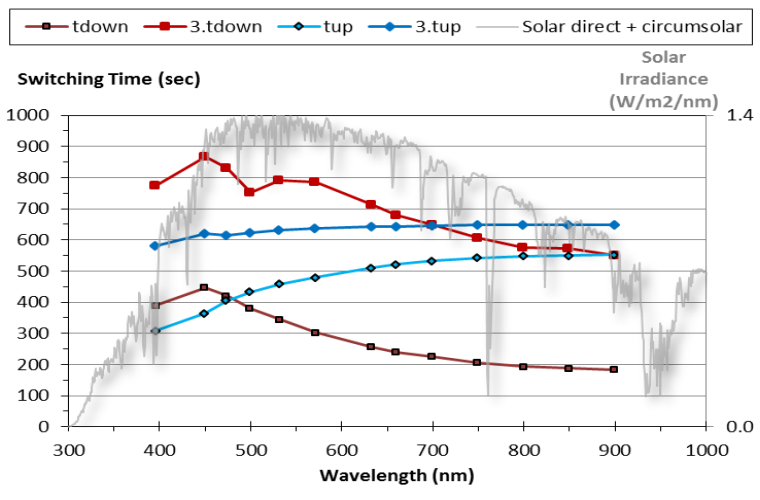

Fig. 15: EC window switching $\mathrm{ON}$ and $\mathrm{OFF}$ times.

$f=\frac{3 \tau_{\text {down }}}{3 \tau_{\text {up }}}$

Fig. 14 compares the up (to transparent state) and down (to opaque state) switching times of the SPD glazing with the solar spectrum. The curves show opposite behaviours affected by a factor $\mathrm{f}$ defined in (eq. 6). In the 400-700nm region the transmission switches from $48.54 \%$ to $1.27 \%$ (Lemarchand, 2014). Simultaneously the "f" factor 
decreased from $\times 4.9$ at $400 \mathrm{~nm}$ to a minimum $\times 1.17$ at $633 \mathrm{~nm}$, before it started strongly picking up in the infrared region. At $900 \mathrm{~nm}$, the switching "down" time was x10.7 longer than the switching "up" time. Particles are forced to align with the electrical fields between the conductive layers when the window is switched ON. Reversely, when turned OFF, the voltage across the SPD film drops to OVac, the electrical field disappears and the particles are free to naturally disperse in random orientations within the Brownian fluid forming the SPD film. It was then expected, as the experiment confirmed, that the switching ON time would be much shorter than the switching OFF time. The switching ON time could not be calculated theoretically due to a lack of information on the particle composition but this one was expected to be in the hundreds of milliseconds - this was also confirmed by the results obtained in Fig. 14. Using Hsu et al. (2005) equations, the theoretical switching OFF time was calculated to be about $1100 \mathrm{~ms}$ or $2000 \mathrm{~ms}$ respectively for ethanol and propanol as the organic fluid considered. In the OFF state the window appeared with a dark blue tint as a direct consequence of the particle rotating and reaching a chaotic order. The time it took for the particles to rotate could then be best visualised in transmission when looking at the blue wavelengths, meaning at wavelengths the particles are efficiently absorptive but with the least absorption. The $\tau_{\text {down }}$ time measured at $450 \mathrm{~nm}$ and $473 \mathrm{~nm}$ were $2100 \mathrm{~ms}$ and $1800 \mathrm{~ms}$ respectively and match with the particle rotation time order of magnitude expected. Strongly absorbed wavelengths have their transmitted intensities reach a minimum even before the particles finish their rotation, consequently reducing the switching OFF time. This appeared in Fig. 14 from 572nm to 700nm with a factor below $x 1.5$. The particles being increasingly transparent as the incident infrared wavelength is increased, the intensity variation tends to zero and the switching OFF time tends to infinite.

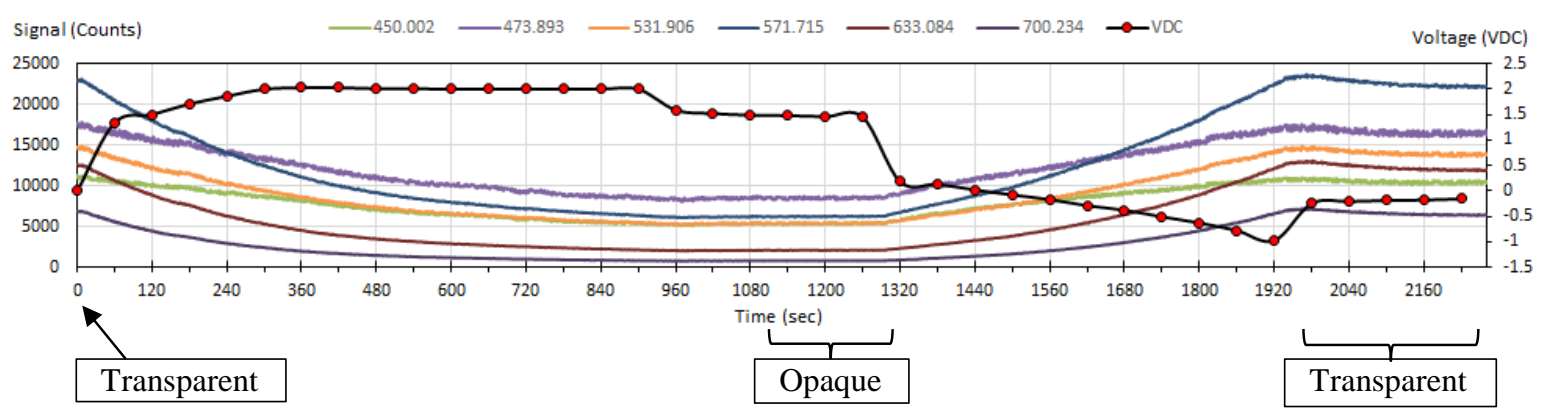

Fig. 16: EC window voltage and transmission variation in time at selected wavelengths.

Fig. 16 illustrates the intensity variation of the transmitted light at specific wavelengths when switched from transparent to opaque and from opaque to transparent. The voltage measured at the window electrodes is plotted on the secondary axis. In the initial transparent state the window was not operated for 7 days prior the experiment and voltage was $0 \mathrm{Vdc}$. When switching off the window, the voltages rises up to $+2 \mathrm{Vdc}$ after $\sim 5$ minutes and remained constant until the windows reached its opaque state with the voltage reduced down to $+1.5 \mathrm{Vdc}$. At this stage, it is believed the window acting as a capacitor was fully "charged" and retained the $+1.5 \mathrm{Vdc}$. Small crystallite systems such as those used in EC windows have been shown to behave more like capacitor than faradaic systems and this may offer an explanation for this apparent discharging behaviour (Ho et al. 1997). A plot of charge versus applied voltage could aid in better understanding this behaviour. Switching the window to the transparent state reduced the voltage to just above $+0 \mathrm{Vdc}$. The voltage continued decreasing to $-1 \mathrm{Vdc}$ when the window reached a maximum transmission. Once the transparent state was reached, the voltage flattened just below $0 \mathrm{Vdc}$. The final transmission state was slightly lower than the initial state but it is believed that, should the transmission and voltage measurements be monitored over a longer period, the transmission would have equalised and the voltage reached 0Vdc.

Fig. 15 shows a $3 \tau_{\text {down }}$ switching times of the EC window from transparent to opaque varying from 867 seconds at $450 \mathrm{~nm}$ down to 549 seconds at $900 \mathrm{~nm}$. Switching from opaque to transparent resulted in curves in the opposite trend. The $\tau_{\text {up }}$ increased from 308 seconds at $400 \mathrm{~nm}$ to 552 seconds at $900 \mathrm{~nm}$. The $3 \tau_{\text {up }}$ switching time to the transparent state varied from 582 seconds to 648 seconds at the respective wavelength. The $3 \tau_{\text {up }}$ values appeared relatively constant across the visible range. A switching speed of this order makes EC windows unsuitable for instantaneous glare control and reactive rule based control (RBC). The ambient temperature at the time of testing was $21^{\circ} \mathrm{C}$ and $\mathrm{EC}$ switching time is known to decrease with an increase in temperature (LBNL, 2006) such as in the warm condition of sunny climates. Whilst this slow switching speed may be seen as a disadvantage, it is worth noting that this slow switching speed would be imperceptible to building occupants. The rapid switching of SPD and PDLC would however be noticeable and may have an impact on occupant comfort as they experience rapid changes of their environment. To mitigate such issues, controllers can adjust the switching speed to control the desired indoor lighting condition. A slow switching EC window, if properly controlled offers the possibility to modulate visible light and 
heat transmittance without any obvious change in the window appearance. A model predictive control (MPC) strategy, with an appropriate time horizon, could offer the possibility of using the slow response time of the EC window combined with expected building conditions over the prediction time horizon to maintain a comfortable occupant environment, primarily by reducing heat gain in the IR range. This type of system is likely to perform better in cooling dominated climates where a constant glare reduction is required rather than a strategic glare control.

Switchable windows need to switch in the visible range as fast as the eye response, i.e. $13 \mathrm{~ms}$ to $80 \mathrm{~ms}$, for the user to have spontaneous control of the glare. Measurements on the SPD glazing showed that $95 \%$ of the full transmission change occurred with switching times of $\sim 0.5 \mathrm{~s}$ and $\sim 1$ second when respectively switching ON and OFF the window. These switching times were consequently 6 to 77 times too long to instantly control glare to the eyes visible response. Moreover, considering a perception of discomfort can be perceived in the order of 1.25 seconds, it would be preferred to have a similar response time in the infrared to instantaneously control solar heat transfer through the switchable glazing. A faster switching speed, achievable with increased absorption from the particles, would then be preferable for wavelengths above $800 \mathrm{~nm}$.

Tab. 4: PDLC switching time.

\begin{tabular}{|r|c|c|c|c|c|c|c|c|c|}
\hline Wavelength (nm) & 378 & 396 & 473 & 532 & 572 & 633 & 660 & 850 & 900 \\
\hline Average Switching OFF time (ms) & 25 & 25 & 30 & 35 & 50 & 45 & 55 & 40 & 45 \\
\hline Average Switching ON time (ms) & 15 & 15 & 15 & 15 & 15 & 15 & 15 & 15 & 15 \\
\hline
\end{tabular}

As shown in Tab. 4, the switching time for PDLC under applied voltage was as low as $15 \mathrm{~ms}$ but is likely to be even faster and wavelength dependent as the recorded values were limited by the spectrometer integration time. For the same window size the SPD required 300-400ms which was at least 20 to 25 times longer. The relaxation time of the LC molecules was at least 2-3 times longer than the switching ON time. Due to their light scattering properties, PDLC windows are primarily applied as indoor privacy walls. The control of glare and thermal response from outdoor conditions is not relevant in that particular case but LC based windows with an embedded dye absorbing the desired wavelength range such as the Licrivision ${ }^{\mathrm{TM}}$ product from Merck (2017) enable control of glare, solar heat transfer and the desired window colour. The fast switching speed of the PDLC windows is noted to be as fast as the human eye response (13ms to $80 \mathrm{~ms})$ and LC based technologies are consequently ideal for glare control.

\section{Conclusion}

The SPD window highly modulated the transmission from $390 \mathrm{~nm}$ to $820 \mathrm{~nm}$ with $51.2 \%$ transmission over the visible range in the transparent state. It also showed a strong absorption and low modulation efficiency in the infrared range reaching below $30 \%$ efficiency from above $1000 \mathrm{~nm}$ and below $10 \%$ above $1350 \mathrm{~nm}$. These results led to $49.6 \%$, $32.8 \%$ and only $1.1 \%$ of the solar power dynamically controllable respectively in the visible, near-infrared and the short-wave infrared wavelength range. Using the simulation results of a glazing formed of three $4 \mathrm{~mm}$ thick glass panes and two EVA layers $0.2 \mathrm{~mm}$ thick, improving on the SPD film dynamic absorption efficiency by $16.8 \%$ within the near-infrared range could lead to additional modulation of up to $51.2 \%$ of the transmitted solar energy within the $780 \mathrm{~nm}-1400 \mathrm{~nm}$ range. This is equivalent to an additional modulation of the solar energy transmitted from $4 \%$ to $>5 \%$ according to the clearness of the glass used. The switching time was shown to be strongly dependent on the wavelength. Switching to the transparent state, the $3 \tau$ switching time varies from $200 \mathrm{~ms}$ to $400 \mathrm{~ms}$ but, most importantly varies from $450 \mathrm{~ms}$ to $2000 \mathrm{~ms}$ when switching the window to the opaque state. The measured $1800 \mathrm{~ms}$ switching time to the opaque state at $473 \mathrm{~nm}$ is in concordance with the theory. In the visible range, the $3 \tau$ switching time of the SPD window is in average 4 to 66 times slower than the human eye response. Increasing the switching speed in the visible range would further help instantaneous glare control. To match the estimated 1.25 seconds required for a human to feel some discomfort from instantaneous solar heat transfer variation due to the passing of clouds, engineering windows with a faster and stronger modulation over a wider wavelength range starting at 800nm is preferable. Scattering effects were low for SPD windows but were the major physical effect of PDLC windows and indicated the need to specify the distance between the detector and the window when referencing optical transmission. The switching time to the transparent state of the PDLC was below $15 \mathrm{~ms}$ but also varies from $25 \mathrm{~ms}$ to $55 \mathrm{~ms}$ according to the wavelength when turning back the window to its opaque mode. In both case this switching time is sufficiently fast to control glare but, due to its milky-white appearance, is most appropriate for privacy rather than façade glazings. However, a UV filter with a wavelength cut-off at 380nm would provide additional protection where both EVA and PMMA are highly transparent and consequently extend the service lifetime of building integrated switchable windows and protect from the degradation (colour fading) of indoor materials. The switching 
time of the EC window appeared particularly dependant to the wavelength when switching the window to the opaque state while it appeared relatively uniform when switching to the transparent state. It should be noted that these times may be expected to decrease with an increase in temperatures above the $21^{\circ} \mathrm{C}$ measured during testing and with an increased exposure to sunlight. These switching times are slow compared to SPD and PDLC windows and for this reason they should not be subject to the same control methodologies. Switching times of this order make them unsuitable for instantaneous glare control and better suited to predictive control where heating/cooling or lighting demand can be predicted in advance of the required switching operation. The EC window was able to modulate only $32.8 \%$ of the solar energy within the $380 \mathrm{~nm}-1020 \mathrm{~nm}$ range making it more suited to climates with high solar irradiance. An increase in the transmission levels, particularly in the infra-red range, would make it more suitable in climates that may require greater levels of visible light and solar heat gain.

\section{References}

Albregtsen, F. (2008). Reflection, refraction, diffraction, and scattering. [online] Available from https://www.uio.no/studier/emner/matnat/ifi/INF-GEO4310/h09/undervisningsmateriale/imaging-kap2.pdf [Accessed June 2016].

ASTM - American Society for Testing and Materials (2012). Reference Solar Spectral Irradiance: Air Mass 1.5. [online] Available from http://rredc.nrel.gov/solar/spectra/am1.5/ [Accessed June 2016].

Czanderna, A. W. \& Pern, F. J. (1996). Encapsulation of PV Modules Using Ethylene Vinyl Acetate Copolymer as a Pottant: A Critical Review. Solar Energy Materials and Solar Cells, 43, pp. 101-181.

Jones, L. (2009). "Thermal touch." Scholarpedia 4(5): 7955.

Kenny, L. W. (1998). Part VI - General Hazards, Chapter 42 - Heat and Cold: Physiological responses to the thermal environment, Encyclopedia of Occupational Health and Safety 4th Edition.

Gueymard, C. A. (2007). Advanced Solar Irradiance Model and Procedure for Spectral Solar Heat Gain Calculation. ASHRAE Transactions, 113 (1), pp. 149-164.

Gueymard, C. A. \& duPont, W. C. (2009). Spectral effects on the transmittance, solar heat gain, and performance rating of glazing systems. Solar Energy, 83, pp. 940-953.

Ho, K. et al., 2006. Proceedings of the Third Symposium on Electrochromic Materials, Electrochemical Society.

Hsu, H.-Y., Sharma, N., Ruoff, R. S. \& Patankar, N. A. (2005). Electro-orientation in particle light valves. Nanotechnology 16 (2), pp. 7.

LBNL (Lawrence Berkeley National Laboratory), 2006. PIER Final Project Report: A Design Guide for EarlyMarket Electrochromic Windows. PIER Final Project Report, (May), pp.1-58. Available at: www.energy.ca.gov [Accessed October 15, 2017]

Lemarchand, P, Norton, B \& Doran, J. (2014, Nov 27). 'Smart Switchable Technologies for Glazing and Photovoltaic Applications', Energy Procedia, Volume 57, Pages 1878-1887.

Lewis, T. (2014). New Record for Human Brain: Fastest Time to See an Image. Live Science.

Merck (2017). Liquid Crystal Window Technology. [online] Available from https://www.licrivision.com/en/LCW_solar_control_glazing.html [Accessed Oct. 2017].

NWSWFO (National Weather Service Weather Forecast Office). (2004). "Heat Index." from http://www.nws.noaa.gov/os/heat/heat_index.shtml [Accessed Oct. 2017]

Polyanskiy, M. (2016). Refractive index database. [online] Available from http://refractiveindex.info/ [Accessed June 2016].

Waide, P. A. \& Norton, B. (2003). Variation of Insolation Transmission With Glazing Plane Position and Sky Conditions. ASME Journal of Solar Energy Engineering, 125 (2), pp. 182-189.

Wienold, J. (2010). Daylight Glare in Offices. Fraunhofer-Verlag. 